\title{
Recoding of Semantic and Acoustic Information in Short-term Memory ${ }^{1}$
}

\author{
Glen A. RASER \\ University of Michigan, Ann Arbor, Michigan 48104
}

\begin{abstract}
A probe recognition task requiring either identity, homophone, or synonym recognition was used to test an acoustic-to-semantic recoding hypothesis which had been suggested as an alternative explanation of a previous demonstration of semantic encoding in STM. With synonym recognition, conditions were compared where identical acoustic inputs were provided but in one condition a semantic relationship existed between the probe word and one of the list words while such a relationship did not exist in the other condition. The recoding hypothesis was not supported as the false-alarm rate in the latter condition remained at chance level.
\end{abstract}

Recent studies and speculations concerning short-term memory (STM) have not been entirely consistent with regard to the question of whether or not semantic encoding is possible in STM. Kintsch and Buschke (1969) have argued that encoding in STM is exclusively acoustic as evidenced by a lack of any semantic similarity effects in the last few serial positions in a probe recall study. Baddeley and Dale (1966) also failed to find significant retroactive inhibition effects due to semantic similarity in a one-trial immediate recall task while such effects were found with a multitrial task.

Shulman (1970) reported a probe recognition study which he interpreted as showing semantic encoding in STM when the task required such encoding. The task involved the serial presentation of a list of ten words, then a cue to specify which of three types of recognition judgments $S$ was to make, and then a probe word which may or may not have had one of the specified relationships to one of the words in the list. Subjects were asked to judge whether or not the probe word was a

1 This study was conducted while the author was the recipient of a National Institute of Mental Health Postdoctoral Fellowship and was supported, in part, by Office of Education Research Grant OEG-O-9320447-4194 (010).

Copyright 1972 by Academic Press, Inc. AlI rights of reproduction in any form reserved. repetition (complete identity), a homophone (acoustical identity), or a synonym (semantic identity) of any of the words in the list. As the cue was not given until after the list was presented, $S \mathrm{~s}$ were forced to encode the list in such a way as to make a response based on either acoustic or semantic information.

Shulman found the serial position curves for recognition performance to be of similar shape under the three criteria, with pronounced recency effects in each case. The fact that synonym recognition occurred with above-chance accuracy and that the synonymrecognition serial position curve showed the same recency effects as the serial position curves for homophone and identity recognition led him to conclude that semantic encoding in STM has been demonstrated. The interpretation of this result as demonstrating semantic encoding in STM depends on the assumption that synonym recognition could not be done on the basis of other kinds of stored information. Shulman pointed out and rejected the possibility that the list words could have been stored phonemically and recoded to a semantic form on those trials requiring synonym recognition. This recoding notion would suggest longer reaction times for synonym recognition than for homophone recognition as some additional time for recoding would be re692 
quired. Shulman's reaction-time curves for serial position of the target word in the list with homophone and synonym recognition showed both a larger intercept and greater slope for the latter criterion. He used the intercept difference between homophone and synonym conditions $(188 \mathrm{msec})$ to estimate the time required to recode a single word (the probe) from an acoustic to a semantic representation and compared this estimate with the slope difference between the two conditions $(22 \mathrm{msec})$ which can be interpreted to represent additional retrieval time in the synonym condition. On the basis of this comparison he rejected the notion of recoding at the time of retrieval since there was such a large difference between the two estimates of the recoding times.

However, this is not a completely satisfying argument against the recoding notion for two reasons. First, the above argument against recoding at retrieval depends on assuming that synonym probes are processed exactly like homophone probes to a point where an acoustic representation is obtained and then are recoded to a semantic form. Only then can intercept differences be considered to estimate recoding time. There is no necessary reason to assume that synonym probes are processed in the above manner. Secondly, it is possible that recoding of the list begins as soon as $S$ knows what recognition criterion to use, that is, as soon as the cue is encoded, and thus recoding time would not be completely reflected in the slope of the reaction-time serial position curve. Measuring reaction time from the onset of the probe to the execution of the response as was done by Shulman would not reflect any recoding done between presentation of the cue and the onset of the probe (a 1.3-sec interval).

A less ambiguous test of the recoding notion would be to compare conditions within the synonym-recognition task which provide identical acoustic input but differing semantic input (i.e., pairs of matched word lists, identical except for the target word which is a synonym of the probe word in one case and a homophone of the target word of the matching list in the other case). If $S$ has only an acoustical representation of the presented list, his performance should be identical in the above two conditions. His performance should differ to the extent that his representation includes other than acoustic information.

Thus, the present study was an attempt to replicate Shulman's results and to test the recoding notion by selecting synonym pairs for the synonym-recognition condition such that one pair member always had a homophone. Thus every synonym recognition trial which included a synonym of the probe in the list (e.g., list word-BARE, probe wordNUDE) could be matched with a trial which included the same acoustic input but without the synonym relationship (e.g., list word-BEAR, probe word-NUDE). An additional condition was included to test for the possibility of recoding from a semantic representation to an acoustic representation in the case of homophone recognition by presenting a synonym of a homophone of the probe word in the list (e.g., list word-GAZE, probe word -STAIR) for comparison with the presentation of the homophone of the probe word itself (e.g., list word-STARE, probe wordSTAIR).

\section{METHOD}

The method used was in major respects identical to that used by Shulman (1970) in that on each trial a short list of words was presented serially followed by a cue to indicate the recognition criterion to be used and then by a probe word. Subjects were instructed to respond "yes" if they thought the probe bore the indicated relationship to any of the list words and to respond "no" otherwise. Reaction times were measured from the onset of the probe to $S$ 's response.

\section{Design}

Eight different conditions defined by recognition criterion (identity, homophone, or synonym recognition) and by the actual relationship between the probe word and the list words were included. When identity recognition was required the list could contain either the probe word itself (condition I-I) or all unrelated words (condition $\mathbf{I}-\mathrm{U}$ ). When homophone recognition was required the list could contain either a homophone 
of the probe word (condition $\mathrm{H}-\mathrm{H}$ ), a synonym of a homophone of the probe word (condition $\mathrm{H}-\mathrm{S}$ ), or only unrelated words (condition $\mathrm{H}-\mathrm{U}$ ). When synonym recognition was required the list could contain either a synonym of the probe word (condition $\mathrm{S}-\mathrm{S}$ ), a homophone of a synonym of the probe word (condition $\mathrm{S}-\mathrm{H}$ ) or all unrelated words (condition $\mathrm{S}-\mathrm{U}$ ). In those conditions where there was a specifiable relationship between the probe and the list, the related word appeared equally often in each of the eight serial positions in the list. Rate of presentation of the list words was varied factorially with all the above conditions. The particular levels used were $500 \mathrm{msec}$ per word and $1000 \mathrm{msec}$ per word.

A single session included 36 practice trials and 108 experimental trials. A single trial consisted of the presentation of a warning signal for $1 \mathrm{sec}$, the presentation of an eight-word list at either 500 or $1000 \mathrm{msec}$ per word, the presentation of a cue for a $1-\mathrm{sec}$ interval to indicate the type of recognition to be made (I for identity, $\mathrm{H}$ for homophone, and $\mathrm{S}$ for synonym recognition), and the presentation of a probe word which remained on the display until a response was made. The 108 experimental trials included 36 under each of the three possible recognition criteria and within each criteria half of the trials were presented at each of the two possible presentation rates. The 18 trials within each rate under the identity criterion included eight I-I trials, one for each serial position, and $10 \mathrm{I}-\mathrm{U}$ trials. The 18 trials within each rate under the homophone criterion included eight $\mathrm{H}-\mathrm{H}$ trials and eight $\mathrm{H}-\mathrm{S}$ trials, one for each serial position in each case, and two $\mathrm{H}-\mathrm{U}$ trials. The 18 trials within each rate under the synonym criterion included eight $\mathrm{S}-\mathrm{S}$ trials and eight S-H trials, one for each serial position in each case, and two S-U trials. An initial random assignment of recognition criterion to trial number was made across all 108 trials and held constant for all $S$ s.

\section{Stimulus Materials}

The stimuli included 108 triads of words made up of a base word, a homophone of the base word, and a synonym of the base word which had been generated for an earlier study (Raser, 1970) and 864 filler words selected from the Thorndike and Lorge word book. All words were from three to seven letters in length and contained either one or two syllables. The 108 triads were first divided into three sets of 36 each and assigned to the three recognition criterion conditions such that word-frequency values were about equally distributed among the three sets. A single filler word was then selected for each of the 108 triads to match the base word in terms of first letter, number of letters, and word-frequency value but was unrelated either acoustically or semantically. The remaining filler words were selected without regard to frequency count but with the letter and syllable length restrictions mentioned above from the Thorndike and Lorge list such that the complete set reflected the proportion of initial letters represented in the Thorndike and Lorge list. Seven of these filler words were randomly assigned to each triad with the restriction that no obvious acoustic or semantic similarities exist between any of the words in the triad and any of the seven filler words. An additional restriction on this assignment was that each set of seven contain at least one word that had a homophone. The final result was 108 sets of 11 words each which with the appropriate selection of list words and probe word could be used at any particular condition within the recognition criterion to which it was assigned.

The assignment of the above word sets and recognition criterion to trial number remained constant for all $S$ s but 36 different assignments of the remaining conditions to trial number were made such that each 11-word set was used equally often at each of the 36 specific conditions within its assigned criterion condition.

\section{Subjects}

The $S$ s were 108 students from the Human Performance Center subject pool at the University of Michigan. They were paid $\$ 1.50$ plus a bonus based on their performance for their participation. Three $S$ s were assigned in the order of their appearance at the laboratory to each of the 36 trial sequences.

\section{Procedure}

All stimuli were presented visually at computercontrolled display stations. Recognition responses were made by pressing one of two keys, one of the left and one of the right of the display screen and labeled "no" and "yes," respectively. A pay-off matrix emphasizing accuracy over speed was imposed but no feedback was given during the experimental session.

\section{RESUlts AND Discussion}

Because each $S$ contributed only one observation in those conditions that involved the serial position manipulation, 18 statistical $S$ s were created by combining the data over sets of six individual $S$ s. An initial $5 \times 2 \times 8$ analysis of variance was performed using the number of "yes" responses as the dependent variable. The respective factors were conditions ( $\mathrm{I}-\mathrm{I}, \mathrm{H}-\mathrm{H}, \mathrm{H}-\mathrm{S}, \mathrm{S}-\mathrm{S}$, and $\mathrm{S}-\mathrm{H})$, presentation rate, and serial position. Significant main effects were found for conditions, $F(4,68)=279.28, p<.001$, and serial position, 
$F(7,119)=9.05, p<.01$, but not for presentation rate $F<1$. The only significant interaction was that involving conditions and serial position, $F(28,476)=2.82, \quad p<.01$. This dependent variable is easily transformed into hit rate for conditions $\mathrm{I}-\mathrm{I}, \mathrm{H}-\mathrm{H}$, and $\mathrm{S}-\mathrm{S}$ and into false-alarm rate for conditions $\mathrm{H}-\mathrm{S}$ and $\mathrm{S}-\mathrm{H}$. The Condition $\times$ Serial Position interaction is presented in this manner in Figure 1 along with the false-alarm rates for the three unrelated conditions. There are several things to note in Figure 1. First, the top three curves

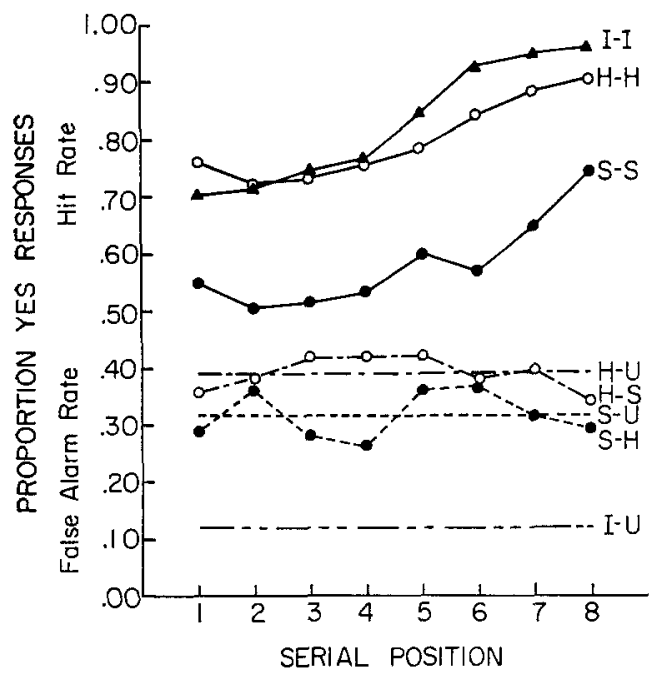

FIG. 1. Proportion of "yes" responses as a function ? of serial position for conditions I-I, H-H, H-S, S-S, and $\mathrm{S}-\mathrm{H}$ and as a function of criterion for the unrelated conditions.

which replicate Shulman's conditions (I-I, $\mathrm{H}-\mathrm{H}$, and $\mathrm{S}-\mathrm{S}$ ) are, for the most part, in accord with his findings. Identity recognition is highest, followed closely by homophone recognition, and at a somewhat lower level by synonym recognition. Also the three curves have highly similar shapes with heightened recency portions in all three cases. In the same analysis of variance with only these three conditions the Condition $\times$ Serial Position interaction failed to reach an acceptable level of significance $F(14,328)=1.04, p>.10$. Thus the significant interaction in the first analysis resulted from including the control conditions which show no consistent variation with serial position rather than from variation in the shape of the serial position curves when an appropriately related word actually appeared in the list.

In addition, false-alarm rate varies as a function of recognition criterion in about the same manner as in Shulman's study with his slower presentation rates. False-alarm rate is lowest for identity recognition and much higher for homophone and synonym recognition.

Although presentation rate did not significantly affect hit rates, $d^{\prime}$ scores of the six rate by criterion conditions showed a pattern of results consistent with those obtained by Shulman. The $d^{\prime}$ measures for the fast and slow presentation rate, respectively, were 2.142 and 2.041 for the identity criterion, 1.112 and 1.131 for the homophone criterion, and .481 and .695 for the synonym criterion. None of these rate differences within a recognition criterion are significant but synonym-recognition performance does show a slight increase with the slower presentation rate while identity recognition and homophone recognition show either no change or a slight decline with the slower rate. Thus, Shulman's presentation rate results are not strongly supported but, on the other hand, are not contradicted either.

Reaction times for correct "yes" responses as a function of serial position were also examined for comparison with Shulman's results. However the serial position functions showed considerable deviation from linearity and were not affected by the presentation rate manipulation. Thus no attempt was made to compare slope and intercept values for bestfitting straight lines.

Although the overall level of performance was somewhat poorer in the present study and reaction times were less regular, the pattern of results shows enough consistency with Shulman's results to suggest that both sets of $S$ s were behaving in about the same way.

Comparisons of the false-alarm rates for the two control conditions $(\mathrm{H}-\mathrm{S}$ and $\mathrm{S}-\mathrm{H})$ and 
the corresponding unrelated conditions are of interest in evaluating the recoding notion. To the extent that acoustic information was stored and recoded to allow a semantic judgment, the false-alarm rate for condition $\mathrm{S}-\mathrm{H}$ should be higher than for condition S-U. This effect should be greatest at the later serial positions where STM responding would be most likely. Also to the extent that semantic information was stored and recoded for making an acoustic judgment, the false-alarm rate for condition $\mathrm{H}-\mathrm{S}$ should be higher than condition $\mathrm{H}-\mathrm{U}$. When serial position is ignored, the control-condition false-alarm rates are at about the same level as the unrelated conditions (.387 and .389 for $\mathrm{H}-\mathrm{S}$ and $\mathrm{H}-\mathrm{U}$, respectively, and .315 and .359 for $\mathrm{S}-\mathrm{H}$ and $\mathrm{S}-\mathrm{U}$, respectively). A $2 \times 2 \times 2$ analysis of variance using the false-alarm proportions with an arcsine transformation as the dependent variable was performed with the factors being presentation rate, recognition criterion (synonym or homophone), and relationship between the probe and the list words (unrelated or the second-order control-condition relationship). The only significant main effect was that of recognition criterion, $F(1,136)$ $=5.41, y<.05$, with a higher false-alarm rate being associated with the homophone criterion. None of the interaction effects reached an acceptable level of significance. Furthermore, as shown in Figure 1, neither of the two control conditions showed any consistent variation with serial position of the related item in the list. Thus there is no evidence from the false-alarm data to support the notion of recoding of information in STM from either an acoustic form to a semantic form or from a semantic form to an acoustic form.

Analysis of reaction-time data for correct rejections, that is, giving a "no" response when a "no" response was appropriate, also are of interest in examining potential confusion effects of the second-order relationships which define the control conditions. Again a $2 \times 2 \times 2$ analysis of variance was carried out with the same factors used in the immediately preceding analysis. The dependent variable was the mean correct rejection reaction time computed for each of the eight conditions after combining the 108 individual $S$ s into 12 sets each containing 9 Ss. The analysis revealed only one effect of even marginal significance, that being the Relatedness $\times$ Criterion interaction, $F(1,88)$. $=3.43, .05<p<.10$. This interaction resulted from correct negative responses having longer reaction times in condition $\mathrm{S}-\mathrm{H}$ than in condition $\mathrm{S}-\mathrm{U}$ while negative responses for conditions $\mathrm{H}-\mathrm{S}$ and $\mathrm{H}-\mathrm{U}$ took about the same amount of time. Thus when $S$ s are attempting synonym recognition, including a word in the list which is acoustically identical to a synonym of the probe word appears to increase the amount of time taken to reject the probe word as being synonymous with any of the list words but does not affect recognition accuracy. With homophone recognition neither speed nor accuracy are affected by including a list word which is semantically similar to a homophone of the probe word.

The recoding notion predicts accuracy differences for the above comparisons and thus may be rejected on the basis of the above data. These findings taken together with the now replicated finding of parallel serial position curves for identity, homophone, and synonym recognition and with Shulman's recent demonstration of semantic confusion effects in STM (Shulman, 1972) provide considerable evidence for semantic encoding in STM. The reactiontime data described above suggest that acoustic information is not completely ignored even when synonym judgments are required, but the process by which such acoustic information affects synonym-recognition reaction times is not at all clear.

\section{REFERENCES}

Badpeley, A. D., \& Dale, H. C. A. The effect of semantic similarity on retroactive interference in long- and short-term memory. Journal of Verbal Learning and Verbal Behavior, 1966, 5, 417-420. 
Kintsch, W., \& BuschKe, H. Homophones and synonyms in short-term memory. Journal of Experimental Psychology, 1969, 80, 403-407.

RASER, G. A. Encoding processes in recognition memory. Unpublished doctoral dissertation, Iowa State University, 1970.

Shulmav, H. G. Encoding and retention of semantic and phonemic information in short-term memory.
Journal of Verbal Learning and Verbal Behavior, 1970, 9, 499-508.

Shulman, H. G. Semantic confusion errors in shortterm memory. Journal of Verbal Learning and Verbal Behavior, 1972, 11, 221-227.

(Received February 8, 1972) 